\title{
Chemokine Ligand 5 (CCL5) and chemokine receptor (CCR5) genetic variants and prostate cancer risk among men of African Descent: a case-control study
}

LaCreis R Kidd ${ }^{1 *}$, Dominique Z Jones ${ }^{1}$, Erica N Rogers ${ }^{1}$, Nayla C Kidd ${ }^{1 *}$, Sydney Beache ${ }^{1}$, James E Rudd ${ }^{2}$, Camille Ragin ${ }^{3}$, Maria Jackson ${ }^{4}$, Norma McFarlane-Anderson ${ }^{4}$, Marshall Tulloch-Reid ${ }^{4}$, Seian Morrison ${ }^{4}$, Guy N Brock ${ }^{5}$, Shirish S Barve ${ }^{1,6}$ and Kevin S Kimbro ${ }^{2}$

\begin{abstract}
Background: Chemokine and chemokine receptors play an essential role in tumorigenesis. Although chemokine-associated single nucleotide polymorphisms (SNPs) are associated with various cancers, their impact on prostate cancer (PCA) among men of African descent is unknown. Consequently, this study evaluated 43 chemokine-associated SNPs in relation to PCA risk. We hypothesized inheritance of variant chemokine-associated alleles may lead to alterations in PCA susceptibility, presumably due to variations in antitumor immune responses.

Methods: Sequence variants were evaluated in germ-line DNA samples from 814 African-American and Jamaican men (279 PCA cases and 535 controls) using Illumina's Goldengate genotyping system.

Results: Inheritance of CCL5 rs2107538 (AA, GA+AA) and rs3817655 (AA, AG, AG+AA) genotypes were linked with a 34-48\% reduction in PCA risk. Additionally, the recessive and dominant models for CCR5 rs1799988 and CCR7 rs3136685 were associated with a 1.52-1.73 fold increase in PCA risk. Upon stratification, only CCL5 rs3817655 and CCR7 rs3136685 remained significant for the Jamaican and U.S. subgroups, respectively.

Conclusions: In summary, CCL5 (rs2107538, rs3817655) and CCR5 (rs1799988) sequence variants significantly modified PCA susceptibility among men of African descent, even after adjusting for age and multiple comparisons. Our findings are only suggestive and require further evaluation and validation in relation to prostate cancer risk and ultimately disease progression, biochemical/disease recurrence and mortality in larger high-risk subgroups. Such efforts will help to identify genetic markers capable of explaining disproportionately high prostate cancer incidence, mortality, and morbidity rates among men of African descent.
\end{abstract}

Keywords: Prostate cancer, Chemokines, Chemokine receptors, Chemokine ligand 5, Chemokine receptor 5, Single nucleotide polymorphisms, Men of African descent

\footnotetext{
* Correspondence: Irkidd01@louisville.edu; nkidd@thacher.org

${ }^{1}$ Department of Pharmacology \& Toxicology, University of Louisville Clinical \&

Translational Research Building, 505 South Hancock Street Room 306, Louisville, KY 40202, USA

Full list of author information is available at the end of the article
} 


\section{Background}

Prostate cancer (PCA) is one of the leading causes of cancer and cancer-related deaths among men in the U.S. and Caribbean and its disparities are well documented [1-3]. From 2003-2007, the average annual PCA incidence rates (229.4 per 100,000) and mortality (54.2 per 100,000) rates among African-American men were 1.6and 2.4-fold higher than US Caucasian men, respectively. According to the 2008 World age-standardized death rates for Caribbean men $(26.3 / 100,000)$ were 2.65 fold higher than all U.S. men $(9.9 / 100,000)$ [3]. Despite its public health burden, the etiology of PCA is not completely elucidated. Established risk factors for PCA include older age, black race, and family history of the disease. Other plausible contributors of prostate tumorigenesis include endocrine, lifestyle, environmental, and genetic factors as well as imbalances in inflammatory, immune surveillance, and angiogenesis pathways.

Among the inflammatory factors, chemokines (e.g., $\mathrm{CC}, \mathrm{CXC}, \mathrm{XCL}$, and $\mathrm{C}-\mathrm{X} 3-\mathrm{C}$ ) play a pivotal role in chemotaxis, leukocyte trafficking, lymphocyte development, angiogenesis, host response to infection, inflammatory processes, tumor development, migration and metastasis. Chemokines mediate their actions through $7-$ transmembrane, G protein coupled receptors and serve three major physiological functions. First, they play fundamental roles in the maturation, homeostasis and function of the immune system, as well as facilitate the trafficking of memory $\mathrm{T}$ cells, lymphocytes, monocytes, and neutrophils to the inflammatory site. Secondly, they display chemotactic activity for lymphocytes, monocytes, and neutrophils. Lastly, they attract cancer cells and chemokine receptor bearing cells and have effects on endothelial cells involved in angiogenesis regulation. Several CXC chemokines are potent angiogenesis promoters (i.e., CXCL1, 2, 3, 5, 6, 7) [4-6]; whereas, others inhibit angiogenesis (i.e., CXCL4, 9, 10, 11) [7].

Genetic alterations and altered expression of chemokines and their receptors have been linked to the susceptibility, development and survival of numerous cancers, including prostate cancer [8-12]. In fact, over expression of CCL5 and CCR5 has been detected in prostate tissue and associated with aggressive disease, presumably by triggering leukocyte production and promoting cell survival, proliferation, invasion and metastasis [13-15]. Coding and regulatory regions of a number of chemokine-associated genes directly influence chemokine production and have been demonstrated to modulate the risk of developing various cancers [10,16-21]. The CCL5 -403A allele conferred an increased risk of prostate, oral and pancreatic cancer $[18,22,23]$. Furthermore, inheritance of the CCR7 rs3136685 AG+AA or CCR7 rs3136687 (AG, AG+AA) genotypes was associated with a $60-62 \%$ reduction in multiple myeloma (MM) and chronic lymphocytic leukemia, respectively $[19,21]$. Unfortunately, these previously mentioned studies focused on single SNPs in relation to cancer primarily among men of European descent. Consequently, the influence of individual CCL5, CCR5, CCR7 and other chemokine associated sequence variants on PCA is unknown among men of African descent.

Thus, the current study evaluated main effects of 43 chemokine-related sequence variants in relation to prostate cancer susceptibility among men of African Descent from the U.S. and Jamaica. Preliminary findings from this study will guide future studies that seek to find important diagnostic, tumor classification and prognostication indicators of PCA in high-risk subgroups.

\section{Methods}

\section{Study population}

The current study consisted of 279 cases and 535 controls obtained from two independent case control study sets, as summarized in Table 1, Additional file 1, and Additional file 2. These studies include the Prostate Cancer Clinical Outcome Study $\left(\mathrm{PC}^{2} \mathrm{OS}\right)$ at the University of Louisville and the Prostate Cancer Study in Jamaica at the University of the West Indies, Mona Campus. For the $\mathrm{PC}^{2} \mathrm{OS}$ study, 603 unrelated male residents were recruited from the Washington, D.C. and Columbia, SC areas through the Howard University Hospital (HUH) Division of Urology or related PCA screening programs between 2001 and 2005. This population of men of African descent (i.e., self-reported African Americans, East African Americans, West African Americans, and Afro-Caribbean Americans) consisted of 170 incident PCA cases and 433 controls (Additional file 1). Between March 2005 and July 2007, two hundred twenty-one unrelated Jamaican men were recruited and consecutively enrolled into the prostate cancer case-control study (109 prostate cancer cases, 102 controls) during their first time visit at urology clinics (Additional file 2). Details on case and control ascertainment and inclusion criteria for both subpopulations have ben detailed elsewhere [24,25]. All study participants provided written informed consent for participation in genetic analysis studies under a protocol approved by the Howard University, the HUH Division of Urology, and the University of Louisville Institutional Review Board (\#201.07).

\section{Criteria for chemokine and chemokine receptor gene and SNP selection}

Chemokine-associated genes and SNPs were selected using one or more of following criteria: (1) epidemiological or molecular biological evidence from published reports indicating a relationship between the $\mathrm{SNP} / g e n e$ 
Table 1 Baseline Characteristics among men of African Descent from the US \& Jamaica

\begin{tabular}{|c|c|c|c|}
\hline Characteristics & Cases & Controls & $p_{\text {value }}^{t}$ \\
\hline Number of Participants, $n$ & 279 & 535 & - \\
\hline $\begin{array}{l}\text { Age at enrollment } \\
\text { (yrs), Median (range) }\end{array}$ & $67(45-91)$ & $53(27-89)$ & $<0.0001$ \\
\hline \multicolumn{4}{|l|}{$\begin{array}{l}\text { Family History of } \\
\text { Prostate Cancer, n (\%) }\end{array}$} \\
\hline Yes & $35(16.1)$ & $21(12.5)$ & 0.316 \\
\hline No & $182(83.9)$ & $147(87.5)$ & \\
\hline Missing & $62(22.2)$ & $367(68.6)$ & \\
\hline PSA (ng/ml), median & $11.7(0.01-10,000)$ & $0.9(0.0-4.0)$ & $<0.0001$ \\
\hline \multicolumn{4}{|l|}{ PSA (ng/ml), n (\%) } \\
\hline$<4$ & $37(13.8)$ & $517(99.8)$ & $<0.0001$ \\
\hline$\geq 4$ & $231(86.2)$ & $1(0.2)$ & \\
\hline Missing & $11(0.04)$ & $17(0.03)$ & \\
\hline \multicolumn{4}{|l|}{ Gleason Score, n (\%) } \\
\hline 4 & $12(5.6)$ & & \\
\hline 5 & $14(6.5)$ & & \\
\hline 6 & $74(34.2)$ & & \\
\hline 7 & $70(32.4)$ & & \\
\hline 8 & $18(8.3)$ & & \\
\hline 9 & $22(10.2)$ & & \\
\hline 10 & $6(2.8)$ & & \\
\hline Missing & $63(22.6)$ & & \\
\hline Global WAA, mean (SD) & $0.79(0.25-0.94)$ & $0.767(0.25-0.95)$ & 0.107 \\
\hline
\end{tabular}

Abbreviations: PSA, prostate specific antigen; WAA, West African Ancestry; ${ }^{\dagger}$ Differences in family history or PSA frequencies between cases and controls were tested by a Chi-square test of homogeneity; differences in median age (yrs) and Global West African Ancestry between cases and controls were tested using the Wilcoxon sum Rank test.

with cancer or inflammatory/immune response related diseases; (2) commonly studied loci; (3) marked disparities in genotype frequency comparing men of African descent to their Caucasian counterparts (i.e., $\pm 10 \%$ change); (4) evidence demonstrating a link with alterations in mRNA expression/stability or protein expression/structure or function using in silico tools (e.g., SNPinfo) or published reports (http://snpinfo.niehs.nih. gov/snpfunc.htm); and (5) a minor allele frequency $\geq 1 \%$ reported in the National Center for Biotechnology Information (NCBI) Entrez SNP, (www.ncbi.nlm.nih.gov). On average, a majority of the SNPs had minor allele frequencies ranging from $26-27 \%$. However, five SNPs with allele frequencies greater than $1 \%$ but less than $5 \%$ were included in the analysis to explore whether rare SNPs would lead to substantial gains in effect sizes (i.e., 2-3 fold increases in risk) and contribute to missing genetic heritability $[26,27]$. The SNPinfo webserver enabled us to annotate and/or predict the functional consequence of chemokine-associated sequence variants on alternative alleles, as summarized in Additional file 3.

\section{Genetic analysis of variant chemokine-associated SNPs}

In order to evaluate and validate chemokine-related markers as predictors of PCA risk, de-identified germline DNA from PCA cases and disease-free individuals were genotyped using a custom Illumina GoldenGate Genotyping assay with VeraCode Technology and BeadXpress reader, according to the manufacturer's instructions [28].

\section{Ancestry markers}

Cases and controls were also genotyped with a set of 100 genome-wide ancestry informative markers to correct for potential population stratification among our admixed population of self-reported African-Americans, West African-Americans, East African-Americans, AfroCaribbean-Americans, as previously described [29,30]. Study participants were grouped from lowest to highest genetic West African Ancestry, with scores ranging from $0-100 \%$. These 100 markers were assembled using DNA from self-identified African-Americans (Coriell Institute for Medical Research, $\mathrm{n}=96$ ), Yoruban West Africans (HapMap, $\mathrm{n}=60$ ), West Africans (Bantu and Nilo Saharan speakers, $\mathrm{n}=72$ ), Europeans (New York City, $\mathrm{n}=$ 24), and CEPH Europeans (HapMap Panel, $\mathrm{n}=60$ ), as previously reported [30]. Individuals with a West African ancestry (WAA) score $\geq 25 \%$ and available chemokineassociated data were included in the final analysis.

\section{Quality control assurance and data management of genotype data}

At the onset of the project, allelic discrimination focused on chemokine associated SNPs among men of African Descent. To minimize misclassification bias, laboratory technicians were blinded to the case status of study participants. Each batch of up to 96 samples included four non-DNA template controls and eight duplicate samples, enabling us to calculate the percent contamination (i.e., 0\%) and concordance rates (i.e., 100\%) per batch. Genotype call rates were calculated separately for each SNP and study participant. Lastly, we tested whether the distribution of the genotypes among disease-free individuals had significant departures from the HardyWeinberg equilibrium (HWE).

Prior to performing marker statistics, we excluded subjects who had genotype call rates that were $<90 \%$. To ensure high quality data, nine SNPs were excluded from the final analysis if: the distribution of the genotypes among controls deviated substantially from the HardyWeinberg Equilibrium, using a conservative significance level cut-off value of $\mathrm{P} \leq 0.005(\mathrm{n}=1)$; they had a minor allele frequency $<1 \%(\mathrm{n}=6)$; or low genotype call rates $<95 \%(\mathrm{n}=2)$. Following data clean-up, 43 chemokinerelated SNPs were included in the final analysis consisting of 814 men of African descent (279 cases, 535 
controls). All quality control analyses and data management was performed using Golden Helix's SNP Variation Software 7.0 (Bozeman, MT).

\section{Statistical analysis for single gene effects}

Univariate and multivariate analyses were used to evaluate chemokine-associated SNPs among men of African descent in relation to prostate cancer risk. To assess whether inheritance of one or more chemokine allele(s) influence the risk of developing PCA, we tested for significant differences in the distribution of homozygous major, heterozygous, or homozygous minor genotypes between cases and controls using the chi-square test of homogeneity. The association between PCA risk and selected polymorphic genes, expressed as odds ratios (ORs) and corresponding 95\% confidence intervals (CIs), were estimated using unconditional multivariate LR models adjusted for age. LR analyses for genetic variants and PCA development were conducted using the major or common genotype as the referent category. All analyses were conducted using SAS 9.3 (SAS Institute Inc., Cary, NC) and SNP Variation Software 7.0 (GoldenHelix, Bozeman, MT). Statistical significance was assessed using a False Discovery Rate cut-off of 0.05 , in order to adjust for multiple comparisons.

\section{Statistical power for single gene effects}

We conducted calculations to determine the statistical power of our sample to detect significant relationships between chemokine-related genotypes and PCA development. The expected risk estimates of our study can be estimated by specifying values for a number of parameters, including a minor allele frequency of at least 26.5\%, National Cancer Institute's estimate of PCA disease prevalence $(0.74 \%)$, number of cases $(n=279)$, and number of controls $(n=535)$. We assumed the causal SNP was in complete linkage disequilibrium with the predisposing variant $\left(\mathrm{r}^{2}=1.0\right)$. Based on our sample size for the total population, U.S. and Jamaican men, we had $>80 \%$ power to detect genetic markers with odds ratios (ORs) of $\geq 1.5, \geq 1.55$, and $\geq 2.0$ for PCA risk, respectively, for minor allele frequency of at least $26.5 \%$, assuming a co-dominant genetic model with 1 degree of freedom (df). Power calculations were performed using Power for Genetic Association Version 2 Software [31].

\section{Results}

\section{Population description}

The demographic and other pertinent characteristics of cases and controls for the entire study population and each study center are summarized in Table 1, Additional file 1, and Additional file 2. Overall, men diagnosed with prostate cancer were 14 years older and had higher PSA levels than controls $(P<0.0001)$. Disease-free men from
Jamaica were about 9 years older than U.S. controls; however, there were no significant differences in family history of prostate cancer or PSA levels for these disease-free subgroups. Jamaican cases had 7 fold higher PSA levels $(\mathrm{P}<0.0001)$ and slightly higher median Gleason scores $(\mathrm{P}=0.018)$ compared to cases from the U.S. There were no differences in the distribution of family history of prostate cancer comparing: (1) cases to controls from the total population $(\mathrm{P}=0.316)$ (Table 1 ), U.S. alone $(\mathrm{P}=0.592)$ (Additional file 1$)$, or Jamaica alone $(\mathrm{P}=0.272)$ (Additional file 2$)$; and (2) controls $(\mathrm{P}=0.757)$ or cases $(\mathrm{P}=0.830)$ comparing the two study centers (data not shown).

\section{Prevalence of minor alleles/genotype frequency comparing men of African Descent from the U.S. and Jamaica}

Overall, the chemokine-related SNPs were fairly common among disease-free individuals from the entire sub-population of U.S. and Jamaica, with average minor frequencies of $26-27 \%$ and a standard deviation of $14 \%$, respectively (data not shown). Thirty-eight SNPs had minor allele frequencies $\geq 5 \%$. For exploratory purposes, five rare SNPs (CCR9 rs12721497, CCL17 rs11076191, CCL11 rs4795896, CCL21 rs11574916, CXCL12 rs1801157) were analyzed with minor allele frequencies ranging between $1.5-4.9 \%$. The minor allele frequency comparing controls from the U.S. and Jamaica were strongly correlated $\left(R^{2}=0.957\right)$. Only 5 out of the 43 SNPs analyzed were discordant comparing men of African descent from the U.S. to men from Jamaica (P < 0.0487), namely CCL17 rs11076191, CCL21 rs11574916, CCR7 rs3136685, CCR7 rs3136687, and CCR9 rs12721497.

\section{Relationship between chemokine sequence variants and prostate cancer risk}

Among all men of African descent, five sequence variants were significantly associated with the risk of developing prostate cancer, as summarized in Table 2. Possession of the CCL5 rs2107538 GA+AA $\left(\mathrm{OR}_{\text {unadjusted }}=0.59 ; 95 \% \mathrm{CI}=\right.$ $0.44,0.80)$ or $C C L 5$ rs3817655 GA+AA $\left(\mathrm{OR}_{\text {unadjusted }}=\right.$ $0.54 ; 95 \% \mathrm{CI}=0.40,0.74)$ genotype was linked with a $41-46 \%$ reduction in PCA risk in the unadjusted LR models. These effects remained significant for both SNPs after adjusting for age (CCL5 rs2107538: $\left.\mathrm{OR}_{\text {adjusted }}=0.66 ; 95 \% \mathrm{CI}=0.46,0.96\right)$ and CCL5 rs3817655: $\left.\mathrm{OR}_{\text {adjusted }}=0.56 ; 95 \% \mathrm{CI}=0.39,0.81\right)$. The recessive genetic model for CCR5 rs1799988 $\left(\mathrm{OR}_{\text {adjusted }}=\right.$ 1.52 ; $95 \% \mathrm{CI}=1.02,2.26)$ as well as the dominant genetic models for CCR7 rs3136685 $\left(\mathrm{OR}_{\text {adjusted }}=1.66\right.$; $95 \% \mathrm{CI}=$ $1.09,2.54)$ and CCR7 rs3136687 (OR adjusted $=1.14 ; 95 \%$ $\mathrm{CI}=1.12,1.16)$ were associated with a significant 1.14 1.66 fold increase in PCA risk within the age adjusted 
Table 2 Association between chemokine associated SNPs and prostate cancer risk among men of African descent

\begin{tabular}{|c|c|c|c|c|c|c|c|c|c|c|}
\hline Genes & dbSNP ID & $\begin{array}{l}\text { Location } \\
\text { predicted } \\
\text { functiont }\end{array}$ & Genotype & Cases n (\%) & Controls n (\%) & $\begin{array}{l}\text { Unadjusted } \\
\text { OR }(95 \% \mathrm{Cl})\end{array}$ & $\begin{array}{l}\text { Adjusted } \\
\text { OR }(95 \% \mathrm{Cl})\end{array}$ & P-value & P-trend & FDR \\
\hline \multirow[t]{5}{*}{ CCR5 } & rs1799988 & 5'UTR & $\mathrm{AA}$ & 85 (30.7) & $194(36.7)$ & 1.00 (referent) & 1.00 (referent) & 0.005 & 0.0039 & 0.0682 \\
\hline & & TFBS & AG & 107 (38.6) & $227(43.9)$ & $1.08(0.76,1.52)$ & $0.83(0.55,1.25)$ & 0.676 & & \\
\hline & & & GG & 85 (30.7) & $108(20.4)$ & $1.80(1.23,2.63)$ & $1.38(0.87,2.17)$ & 0.003 & & \\
\hline & & & $A G+G G$ & $192(69.3)$ & 335 (63.3) & $1.31(0.96,1.78)$ & $1.01(0.70,1.46)$ & 0.09 & & 0.4823 \\
\hline & & & GG vs $(A A+A G)$ & & & $1.73(1.24,2.40)$ & $1.52(1.02,2.26)$ & 0.0013 & & 0.0494 \\
\hline \multirow[t]{5}{*}{ CCL5 } & rs2107538 & 5 ' near gene & GG & 111 (39.8) & $150(28.1)$ & 1.00 (referent) & 1.00 (referent) & 0.002 & 0.001 & 0.0493 \\
\hline & & TFBS & $\mathrm{GA}$ & $124(44.4)$ & $270(50.6)$ & $0.62(0.45,0.86)$ & $0.72(0.49,1.06)$ & 0.004 & & \\
\hline & & & AA & $44(15.8)$ & $114(21.4)$ & $0.52(0.34,0.80)$ & $0.53(0.32,0.89)$ & 0.003 & & \\
\hline & & & $\mathrm{GA}+\mathrm{AA}$ & $168(60.2)$ & $384(71.9)$ & $0.59(0.44,0.80)$ & $0.66(0.46,0.96)$ & 0.0007 & & 0.015 \\
\hline & & & $A A$ vs $(G G+G A)$ & & & $0.83(0.57,1.20)$ & $0.74(0.47,1.16)$ & 0.057 & & 0.4735 \\
\hline \multirow[t]{5}{*}{ CCL5 } & rs3817655 & Intron 2 & GG & $114(41.0)$ & 147 (27.5) & 1.00 (referent) & 1.00 (referent) & 0.004 & 0.002 & 0.019 \\
\hline & & TFBS & GA & $115(41.4)$ & $278(52.0)$ & $0.53(0.38,0.74)$ & $0.57(0.38,0.84)$ & 0.0002 & & \\
\hline & & & $\mathrm{AA}$ & 49 (17.6) & $110(20.5)$ & $0.57(0.38,0.87)$ & $0.54(0.32,0.89)$ & 0.0009 & & \\
\hline & & & $\mathrm{GA}+\mathrm{AA}$ & $164(59.0)$ & $388(72.5)$ & $0.54(0.40,0.74)$ & $0.56(0.39,0.81)$ & 0.0001 & & 0.0038 \\
\hline & & & $A A$ vs $(G G+G A)$ & & & $0.83(0.57,1.20)$ & $0.74(0.47,1.16)$ & 0.317 & & 1 \\
\hline \multirow[t]{5}{*}{ CCR7 } & rs3136685 & Intron 1 & $\mathrm{AA}$ & 55 (19.7) & $151(28.3)$ & 1.00 (referent) & 1.00 (referent) & 0.029 & 0.031 & 0.3078 \\
\hline & & & $A G$ & $139(49.8)$ & $237(44.4)$ & $1.61(1.11,2.34)$ & $1.86(1.18,2.92)$ & 0.012 & & \\
\hline & & & GG & 85 (30.5) & $146(27.3)$ & $1.60(1.06,2.40)$ & $1.39(0.85,2.28)$ & 0.024 & & \\
\hline & & & $A G+G G$ & $224(80.3)$ & $383(71.7)$ & $1.61(1.13,2.28)$ & $1.66(1.09,2.54)$ & 0.008 & & 0.599 \\
\hline & & & GG vs $(A A+A G)$ & & & $1.16(0.85,1.60)$ & $0.92(0.62,1.35)$ & 0.348 & & 0.1615 \\
\hline \multirow[t]{5}{*}{ CCR7 } & rs3136687 & Intron 1 & $\mathrm{AA}$ & $84(30.1)$ & $173(32.4)$ & 1.00 (referent) & 1.00 (referent) & 0.041 & 0.458 & 0.3523 \\
\hline & & & $A G$ & $153(54.8)$ & $249(46.5)$ & $1.26(0.91,1.76)$ & $1.45(0.97,2.16)$ & 0.161 & & \\
\hline & & & GG & $42(15.1)$ & $113(21.1)$ & $0.77(0.49,1.19)$ & $0.96(0.57,1.62)$ & 0.234 & & \\
\hline & & & $A G+G G$ & $195(69.9)$ & $362(67.6)$ & $1.11(0.81,1.52)$ & $1.14(1.12,1.16)$ & 0.516 & & 0.7159 \\
\hline & & & $\mathrm{GG}$ vs $(\mathrm{AA}+\mathrm{AG})$ & & & $0.66(0.45,0.98)$ & $0.76(0.49,1.20)$ & 0.037 & & 0.5092 \\
\hline
\end{tabular}

LR models. After controlling for multiple comparisons, the dominant genetic models for the two CCL5 SNPs (rs2107538, rs3817655) remained significant with falsediscovery rates $(\mathrm{FDR}) \leq 0.0150$; whereas, the recessive genetic model for CCR5 rs1799988 was marginally significant $(F D R=0.0494)$.

In an exploratory analysis, we evaluated risk estimates for all 43 chemokine targets for each racial/ethnic group, as depicted in Table 3. Among U.S. men, CCR5 rs1799988, CCL5 (rs2107538, rs2280789, rs3817655), CCL25 rs2032887, and CXCR7 rs1045879 were associated with PCA risk. Inheritance of the CCL25 rs2032887 AG+GG $\left(\mathrm{OR}_{\text {unadjusted }}=0.66 ; 95 \% \mathrm{CI}=0.46\right.$, 0.96), CCL5 rs $2107538 \mathrm{GA}+\mathrm{AA}\left(\mathrm{OR}_{\text {unadjusted }}=0.52 ; 95 \%\right.$ $\mathrm{CI}=0.36,0.76), C C L 5 \mathrm{rs} 2280789 \mathrm{AG}+\mathrm{GG}\left(\mathrm{OR}_{\text {unadjusted }}=\right.$ $0.60 ; 95 \% \mathrm{CI}=0.41,0.89)$, and $C C L 5$ rs3817655 GA+AA $\left(\mathrm{OR}_{\text {unadjusted }}=0.46 ; 95 \% \mathrm{CI}=0.32,0.68\right)$ genotypes were significantly associated with a $34-54 \%$ reduction in the risk of developing PCA with chi-square p-values ranging from 0.0001-0.027. Although the magnitude of the reduction in PCA risk for CCL5 rs2107538 GA+AA
$\left(\mathrm{OR}_{\text {unadjusted }}=0.52 ; \mathrm{OR}_{\text {adjusted }}=0.63\right), C C L 5 \mathrm{rs} 3817655$ $\mathrm{GA}+\mathrm{AA}\left(\mathrm{OR}_{\text {unadjusted }}=0.46, \mathrm{OR}_{\text {adjusted }}=0.51\right)$ and $C C L 25$ rs2032887 AG+GG $\left(\mathrm{OR}_{\text {unadjusted }}=0.66, \mathrm{OR}_{\text {adjusted }}=0.68\right)$ genotypes remained practically unchanged after adjusting for age, the findings only remained significant for CCL5 rs33817655 SNP after the adjustment. The 1.54-1.62 fold increase in PCA susceptibility was associated with the CCR5 rs1799988 recessive genetic model $\left(\mathrm{OR}_{\text {unadjusted }}=1.62 ; 95 \% \mathrm{CI}=1.08,2.42\right)$ and the CXCR7 rs1045879 AG+GG genotype $\left(\mathrm{OR}_{\text {unadjusted }}=1.54\right.$; $95 \% \mathrm{CI}=1.07,2.22 ; \mathrm{P}=0.02$ ) was lost in the age adjusted risk models.

In the Jamaican population, there was a two-fold increase in PCA susceptibility associated with the CCR5 rs1799987 AA $\left(\mathrm{OR}_{\text {unadjusted }}=2.18 ; 95 \% \mathrm{CI}=1.04,4.58\right)$, CCR5 rs1799988 GG $\left(\mathrm{OR}_{\text {unadjusted }}=2.25 ; 95 \% \mathrm{CI}=1.08\right.$, 4.71), and CCR7 rs3136685 AG+GG $\left(\mathrm{OR}_{\text {unadjusted }}=2.38\right.$; $95 \% \mathrm{CI}=1.05,5.07)$ genotypes, with corresponding chisquare P-values ranging from 0.02-0.037. Additionally, a $54 \%$ reduction in PCA risk was observed for individuals who possessed the CCR9 rs1488371 CA+AA genotype 
Table 3 Association between chemokine associated SNPs and prostate cancer risk, stratified by racial ethnic group

\begin{tabular}{|c|c|c|c|c|c|c|c|c|c|c|c|}
\hline Genes & dbSNP ID & $\begin{array}{l}\text { Location } \\
\text { predicted } \\
\text { function }\end{array}$ & Genotype & $\begin{array}{c}\text { Unadjusted } \\
\text { OR }(95 \% \mathrm{Cl}) \\
\text { US men }\end{array}$ & $\begin{array}{c}\text { Unadjusted } \\
\text { OR }(95 \% \mathrm{Cl}) \\
\text { Jamaican men }\end{array}$ & $\begin{array}{l}\text { Age-adjusted } \\
\text { OR }(95 \% \mathrm{Cl}) \\
\text { US men }\end{array}$ & $\begin{array}{l}\text { Age-adjusted } \\
\text { OR }(95 \% \mathrm{Cl}) \\
\text { Jamaican men }\end{array}$ & $\begin{array}{l}\text { P-value } \\
\text { US men }\end{array}$ & $\begin{array}{l}P \text { trend } \\
\text { US men }\end{array}$ & $\begin{array}{r}\text { P-vlue } \\
\text { Jamaican men }\end{array}$ & $\begin{array}{r}\text { P-trend } \\
\text { Jamaican men }\end{array}$ \\
\hline \multirow[t]{5}{*}{ CCR5 } & rs1799988 & $5^{\prime} U T R$ & AA & 1.00 (referent) & 1.00 (referent) & 1.00 (referent) & 1.00 (referent) & 0.063 & 0.076 & 0.085 & 0.034 \\
\hline & & TFBS & $A G$ & $0.95(0.62,1.44)$ & $1.26(0.66,2.40)$ & $0.68(0.42,1.12)$ & $1.23(0.60,2.52)$ & 0.808 & & 0.484 & \\
\hline & & & GG & $1.58(0.99,2.49)$ & $2.25(1.08,4.71)$ & $1.06(0.60,1.85)$ & $2.23(0.99,5.00)$ & 0.053 & & 0.031 & \\
\hline & & & $\mathrm{AG}+\mathrm{GG}$ & $1.15(0.79,1.68)$ & $1.56(0.86,2.82)$ & $0.80(0.52,1.26)$ & $1.55(0.81,2.98)$ & 0.453 & & 0.142 & \\
\hline & & & $\begin{array}{l}\text { GG vs } \\
(\mathrm{AA}+\mathrm{AG})\end{array}$ & $1.62(1.08,2.42)$ & $1.96(1.04,3.70)$ & $1.28(0.78,2.12)$ & $1.98(0.98,3.98)$ & 0.02 & & 0.037 & \\
\hline \multirow[t]{5}{*}{ CCL5 } & rs2107538 & 5' near gene & GG & 1.00 (referent) & 1.00 (referent) & 1.00 (referent) & 1.00 (referent) & 0.003 & 0.002 & 0.286 & 0.116 \\
\hline & & TFBS & GA & $0.54(0.36,0.80)$ & $0.75(0.40,1.40)$ & $0.67(0.42,1.08)$ & $0.82(0.40,1.64)$ & 0.003 & & 0.375 & \\
\hline & & & AA & $0.48(0.28,0.82)$ & $0.53(0.24,1.16)$ & $0.53(0.28,1.01)$ & $0.52(0.22,1.22)$ & 0.007 & & 0.116 & \\
\hline & & & $A G+A A$ & $0.52(0.36,0.76)$ & $0.68(0.38,1.23)$ & $0.63(0.40,0.99)$ & $0.72(0.37,1.40)$ & 0.001 & & 0.204 & \\
\hline & & & $\begin{array}{l}\text { AA vs } \\
(\mathrm{GG}+\mathrm{GA}) \\
\end{array}$ & $0.68(0.42,1.11)$ & $0.64(0.32,1.26)$ & $0.66(0.37,1.20)$ & $0.58(0.28,1.24)$ & 0.125 & & 0.192 & \\
\hline \multirow[t]{5}{*}{ CCL5 } & rs2280789 & Intron 1 & $\mathrm{AA}$ & 1.00 (referent) & 1.00 (referent) & 1.00 (referent) & 1.00 (referent) & 0.039 & 0.018 & 0.508 & 0.823 \\
\hline & & TFBS & $A G$ & $0.60(0.40,0.90)$ & $1.20(0.66,2.18)$ & $0.60(0.37,0.97)$ & $1.48(0.76,2.89)$ & 0.015 & & 0.549 & \\
\hline & & & GG & $0.62(0.24,1.56)$ & $0.59(0.18,1.89)$ & $1.00(0.34,2.99)$ & $0.42(0.12,1.46)$ & 0.309 & & 0.373 & \\
\hline & & & $\mathrm{AG}+\mathrm{GG}$ & $0.60(0.41,0.89)$ & $1.06(0.61,1.86)$ & $0.64(0.40,1.01)$ & $1.18(0.63,2.20)$ & 0.011 & & 0.82 & \\
\hline & & & $\begin{array}{l}\text { GG vs } \\
(A A+A G) \\
\end{array}$ & $0.72(0.28,1.82)$ & $0.55(0.18,1.80)$ & $1.14(1.12,1.18)$ & $0.36(0.10,1.27)$ & 0.488 & & 0.313 & \\
\hline \multirow[t]{5}{*}{ CCL5 } & rs3817655 & Intron 2 & GG & 1.00 (referent) & 1.00 (referent) & 1.00 (referent) & 1.00 (referent) & 0 & 0.003 & 0.275 & 0.11 \\
\hline & & TFBS & GA & $0.44(0.29,0.65)$ & $0.72(0.38,1.34)$ & $0.49(0.30,0.80)$ & $0.74(0.36,1.50)$ & $<0.0001$ & & 0.302 & \\
\hline & & & AA & $0.56(0.34,0.92)$ & $0.53(0.24,1.16)$ & $0.54(0.29,1.02)$ & $0.50(0.22,1.20)$ & 0.022 & & 0.115 & \\
\hline & & & $\mathrm{GA}+\mathrm{AA}$ & $0.46(0.32,0.68)$ & $0.66(0.36,1.20)$ & $0.51(0.32,0.80)$ & $0.66(0.34,1.28)$ & $<0.0001$ & & 0.167 & \\
\hline & & & $\begin{array}{l}\text { AA vs } \\
(G G+G A)\end{array}$ & $0.88(0.55,1.40)$ & $0.66(0.34,1.28)$ & $0.80(0.46,1.42)$ & $0.61(0.28,1.27)$ & 0.577 & & 0.22 & \\
\hline \multirow[t]{5}{*}{ CCL25 } & rs2032887 & Exon 3 & $\mathrm{AA}$ & 1.00 (referent) & 1.00 (referent) & 1.00 (referent) & 1.00 (referent) & 0.084 & 0.034 & 0.474 & 0.379 \\
\hline & & ESE or ESS & $A G$ & $0.67(0.46,0.99)$ & $1.42(0.80,2.48)$ & $0.70(0.44,1.11)$ & $1.54(0.82,2.88)$ & 0.404 & & 0.225 & \\
\hline & & nsSNP & GG & $0.61(0.29,1.28)$ & $1.10(0.36,3.38)$ & $0.57(0.22,1.42)$ & $0.84(0.24,2.88)$ & 0.196 & & 0.86 & \\
\hline & & $\begin{array}{l}\text { probably } \\
\text { damaging }\end{array}$ & $A G+G G$ & $0.66,(0.46,0.96)$ & $1.37(0.80,2.36)$ & $0.68(0.44,1.05)$ & $1.42(0.78,2.60)$ & 0.027 & & 0.253 & \\
\hline & & missense $\mathrm{R}>\mathrm{H}$ & $\begin{array}{l}\text { GG vs } \\
(A A+A G)\end{array}$ & $0.71(0.34,1.47)$ & $0.93(0.32,2.75)$ & $0.65(0.26,1.60)$ & $0.68(0.20,2.24)$ & 0.357 & & 0.898 & \\
\hline \multirow[t]{3}{*}{ CCR5 } & rs1799987 & Intron 1 & GG & 1.00 (referent) & 1.00 (referent) & 1.00 (referent) & 1.00 (referent) & 0.197 & 0.951 & 0.107 & 0.041 \\
\hline & & TFBS & GA & $1.29(0.88,1.92)$ & $1.28(0.68,2.44)$ & $0.89(0.56,1.44)$ & $1.25(0.61,2.56)$ & 0.191 & & 0.442 & \\
\hline & & & AA & $0.84(0.48,1.48)$ & $2.18(1.04,4.58)$ & $0.58(0.29,1.14)$ & $2.20(0.98,4.94)$ & 0.558 & & 0.039 & \\
\hline
\end{tabular}


Table 3 Association between chemokine associated SNPs and prostate cancer risk, stratified by racial ethnic group (Continued)

\begin{tabular}{|c|c|c|c|c|c|c|c|c|c|c|c|}
\hline & & & $\mathrm{GA}+\mathrm{AA}$ & $1.18(0.80,1.70)$ & $1.56(0.86,2.82)$ & $0.81(0.52,1.26)$ & $1.55(0.81,2.98)$ & 0.399 & & 0.142 & \\
\hline & & & $\begin{array}{l}\text { AA vs } \\
(G G+G A)\end{array}$ & $0.72(0.44,1.21)$ & $1.88(0.99,3.56)$ & $0.61(0.32,1.15)$ & $1.92(0.96,3.88)$ & 0.222 & & 0.051 & \\
\hline \multirow[t]{5}{*}{ CCR7 } & rs3136685 & Intron 1 & $\mathrm{AA}$ & 1.00 (referent) & 1.00 (referent) & 1.00 (referent) & 1.00 (referent) & 0.381 & 0.731 & 0.103 & 0.086 \\
\hline & & & $A G$ & $1.32(0.86,2.02)$ & $2.24(0.98,5.16)$ & $1.58(0.94,2.66)$ & $2.78(1.09,7.08)$ & 0.197 & & 0.056 & \\
\hline & & & GG & $1.07(0.65,1.76)$ & $2.38(1.02,5.58)$ & $1.02(0.56,1.86)$ & $2.52(0.97,6.52)$ & 0.778 & & 0.045 & \\
\hline & & & $\mathrm{AG}+\mathrm{GG}$ & $1.23(0.82,1.84)$ & $2.3(1.05,5.07)$ & $1.36(0.83,2.21)$ & $2.66(1.10,6.42)$ & 0.305 & & 0.037 & \\
\hline & & & $\begin{array}{l}\text { GG vs } \\
(A A+A G)\end{array}$ & $0.90(0.59,1.36)$ & $1.28(0.74,2.25)$ & $0.76(0.46,1.26)$ & $1.16(0.62,2.15)$ & 0.622 & & 0.372 & \\
\hline \multirow[t]{5}{*}{ CCR7 } & rs3136687 & Intron 1 & AA & 1.00 (referent) & 1.00 (referent) & 1.00 (referent) & 1.00 (referent) & 0.022 & 0.294 & 0.229 & 0.218 \\
\hline & & & AG & $1.42(0.89,2.24)$ & $2.16(0.84,5.56)$ & $1.47(0.85,2.54)$ & $1.52(0.53,4.38)$ & 0.133 & & 0.11 & \\
\hline & & & GG & $0.78(0.46,1.34)$ & $2.20(0.84,5.69)$ & $0.85(0.45,1.59)$ & $1.34(0.46,3.96)$ & 0.38 & & 0.106 & \\
\hline & & & $\mathrm{AG}+\mathrm{GG}$ & $1.17(0.76,1.81)$ & $2.18(0.88,5.38)$ & $1.22(0.72,2.05)$ & $1.44(0.52,3.98)$ & 0.482 & & 0.092 & \\
\hline & & & GG vs $(A A+A G)$ & $0.61(0.40,0.93)$ & $1.17(0.68,2.02)$ & $1.14(1.12,1.18)$ & $0.94(0.51,1.75)$ & 0.023 & & 0.573 & \\
\hline \multirow[t]{5}{*}{ CCR9 } & rs1488371 & Intron 2 & $\mathrm{CC}$ & 1.00 (referent) & 1.00 (referent) & 1.00 (referent) & 1.00 (referent) & 0.013 & 0.902 & 0.003 & 0.018 \\
\hline & & & CA & $0.94(0.62,1.40)$ & $0.52(0.26,1.08)$ & $0.88(0.54,1.42)$ & $0.60(0.26,1.31)$ & 0.747 & & 0.08 & \\
\hline & & & $\mathrm{AA}$ & $1.57(0.50,4.90)$ & - & $0.92(0.22,3.80)$ & - & 0.435 & & 0.984 & \\
\hline & & & $C A+A A$ & $0.98(0.66,1.44)$ & $0.46(0.23,0.94)$ & $0.88(0.54,1.40)$ & $0.48(0.22,1.04)$ & 0.901 & & 0.034 & \\
\hline & & & $A A$ vs $(C C+C A)$ & $1.60(0.52,4.97)$ & - & $0.95(0.23,4.00)$ & - & 0.414 & & 0.984 & \\
\hline \multirow[t]{5}{*}{ CXCR7 } & rs1045879 & Exon 1 & AA & 1.00 (referent) & 1.00 (referent) & 1.00 (referent) & 1.00 (referent) & 0.065 & 0.038 & 0.459 & 0.762 \\
\hline & & synSNP & $A G$ & $1.54(1.06,2.26)$ & $1.38(0.77,2.48)$ & $1.30(0.82,2.04)$ & $1.30(0.68,2.50)$ & 0.025 & & 0.279 & \\
\hline & & $L>L$ & GG & $1.53(0.84,2.79)$ & $0.86(0.34,2.16)$ & $1.02(0.47,2.20)$ & $0.74(0.27,2.04)$ & 0.166 & & 0.756 & \\
\hline & & & $A G+G G$ & $1.54(1.07,2.22)$ & $1.24(0.72,2.14)$ & $1.24(0.80,1.93)$ & $1.15(0.62,2.10)$ & 0.02 & & 0.431 & \\
\hline & & & GG vs (AA+AG) & $1.21(0.69,2.13)$ & $0.76(0.31,1.84)$ & $0.88(0.42,1.84)$ & $0.67(0.25,1.80)$ & 0.499 & & 0.538 & \\
\hline
\end{tabular}

Abbreviations: UTR, untranslated region; TFBS, transcription factor binding site; ESE, exonic splicing enhancers ; ESS, exonic splicing silencers; nsSNP, non-synonymous coding SNP; synSNP, synonymous SNP. 
$\left(\mathrm{OR}_{\text {unadjusted }}=0.46 ; 95 \% \mathrm{CI}=0.23,0.94\right)$. Out of the 4 markers, the CCR7 rs3136685 SNP remained significant after adjusting for age. Notably, the magnitude of PCA risk estimates did not change for the CCR5, CCL5, and CCR9 SNPs, comparing the adjusted and unadjusted risk models.

\section{Discussion}

Chemotaxis is an important process required for tumor growth and metastasis. It is regulated by a complex network of chemokines, chemokine receptors and downstream targets that synergistically regulate immune and inflammatory responses. Recent molecular studies have demonstrated that over expression of selected chemokines and chemokine receptors are related to aggressive cancer phenotypes, including lung, breast and prostate cancer. Some observational studies suggest inheritance of susceptibilities detected in chemokine associated genes may alter the risk of developing cancer. However, to our knowledge, there are no published reports on the impact of inheriting multiple functional variants in relation to prostate cancer among men of African Descent. Therefore, the current study evaluated the individual effects of 43 chemokine associated sequence variants on PCA risk among 279 cases and 535 disease-free men of African descent from the U.S and Jamaica. Five SNPs detected in CCL5, CCR5 and CCR7 were significantly associated with prostate cancer risk among all study participants; however, only three markers survived adjustments for potential confounders and multiple hypothesis testing. Notably, inheritance of at least one CCL5 rs3817655 A or CCL5 rs2107538 A loci was linked with a $34-44 \%$ decrease in PCA susceptibility among all men of African Descent. In addition, the recessive genetic model for CCR5 rs1799988 was associated with a 52$73 \%$ increase in PCA risk. We also observed significant main effects for the CCL5 rs3817655 and CCR7 rs3136685 SNPs among U.S. and Jamaican men, respectively. Further discussion will be restricted to the more robust SNPs detected in CCL5 and CCR5.

Several cancer cells, including PCA cells, express chemokines and their cell surface bound receptors. Chemokine ligand 5 (RANTES), is a small molecule with a strong capacity to induce cellular migration of inflammatory cells and production of its receptor (CCR5) in human PCA cell lines [13,32]. The CCL5/CCR5 axis induces PCA cell proliferation and cell invasion. It's speculated that once CCL5 binds to CCR5, it serves as an autocrine factor and activates cellular responses involved in cancer progression [13]. In the current study, possession of the CCL5 rs3817655 A or CCL5 rs2107538 A loci was linked with a protective effect in relation to prostate cancer risk among all men of African descent from the U.S. and Jamaica combined. The directionality of the risk estimates persisted when we stratified by racial/ethnic group; however, the findings were only statistically significant for the U.S. men. In addition, findings for the total and U.S. subgroups remained significant even after adjusting for age and multiple hypothesis testing. The observed protective effects associated with the two aforementioned CCL5 SNPs (rs3817655, rs2107538) may be attributed to a reduction in transcriptional activation, reduced protein levels, and ultimately reduced tumorigenic capacity. To our knowledge, there are no published reports on the impact of the CCL5 rs3817655 SNP on prostate cancer susceptibility or its functional consequence on genes/proteins. On the other hand, the CCL5 rs2107538 G-403A promoter SNP is associated with a decrease in protein expression detected in serum collected from Type II diabetic and disease-free subjects [16]. In addition, this locus has been evaluated in two independent prostate cancer studies. In a study involving 607 Caucasian male residents of Spain (297 cases, 3011 controls), Saenz-Lopez and co-workers (2008) observed a 1.44-fold increase in PCA risk among carriers of the CCL5 rs2107538 GA+AA genotype $(\mathrm{P}=0.039)$ [18]. However, this finding did not corroborate with a larger null report consisting of 1553 Caucasian men (i.e., 815 PCA cases, 738 controls) from Australia [33]. Our findings of a protective effect against PCA among our study participants is consistent with other published reports that reveal a decrease in the risk of developing gastric cancer, lymphoma, and type 1 diabetes $[10,16,20]$. Within a multi-ethnic pancreatic case-control study, the prevalence of the "A" allele was more frequent among disease-free Asian and African-Americans relative to pancreatic cases [23]. However, this allele was more prevalent among Caucasian pancreatic cases relative to controls. Collectively, these findings may suggest inheritance of genetic susceptibilities detected in the CCL5 gene may vary across different racial/ethnic groups.

The functional impact of CCL5 sequence variants is complicated by the high degree of linkage disequilibrium within both the promoter and intron 1 region. An and co-workers (2006), evaluated the impact of three SNPs detected in the promoter region (rs2280788 -28C/G, rs2107538 -405 G/A) and intron 1 of CCL5 rs2280789 [17]. They demonstrated that transcriptional regulation of CCL5 was primarily governed by an intron $1.1 \mathrm{~A} / \mathrm{G}$ SNP (rs2280789). Intron 1.1 G allele corresponded with a strong decrease in transcriptional activity of RANTES; whereas, the $-28 \mathrm{G}$ allele had a modest up-regulation in human cell lines. In our stratified analysis, the intron 1.1 CCL5 rs2280789 AG or AG+GG genotypes were associated with a marginal $36-41 \%$ decrease in PCA risk among men of African descent from the US; however, these findings require further evaluation in a larger study set. The CCL5 rs2280788-28 C/G SNP was not evaluated 
in the current study, since the $\mathrm{C}$ allele frequency is $0 \%$ for African-American men, as reported in NCBI. The functional consequence of CCL5 SNPs is further perplexed by their interaction with downstream receptors.

The biological activity mediated by CCL 5 is facilitated through its interaction with chemokine receptors (CCR1, CCR2, CCR3, and CCR5). However, relative to CCR1-3, CCR5 plays a more important role in CCL5-mediated cell migration [34]. CCR5, a member of the beta chemokine receptor family, is a seven transmembrane protein, which is expressed by $\mathrm{T}$ cells and macrophages. Over expression of CCR5 has been detected in aggressive prostate cancer tissue relative to benign prostatic hyperplasia [32]. The CCR5 rs $1799988 \mathrm{C}$ allele is significantly associated with viral load set point (i.e., decreased time from asymptomatic $\mathrm{HIV}^{+}$to AIDS and increased infectiousness) and AIDS progression [35]. However, there are no published reports for this 5'UTR SNP in relation to prostate cancer or other inflammatory/immune responserelated diseases. In the current study, we observed a 1.52-1.75 fold increase in the risk of developing prostate cancer among all men of African descent who possessed the CCR5 rs1799988 CC genotype. However, the impact of this SNP in relation to PCA risk was more pronounced among men of African descent from Jamaica relative to U.S. men. This increased risk may have an impact on transcriptional activity, which may result in increased protein levels of CCR5; however, this requires confirmation using ex vivo, in vitro, and micro-dissected tumor tissue-based assays.

The strengths, limitations and future directions of this study were considered. Forty-three sequence variants were evaluated in relation to prostate cancer risk among men of African descent from the U.S. and Jamaica. A strong correlation between the minor allele frequencies between these two study populations enabled us to pool genetic data to identify relationships that would have remained undetected if we evaluated the populations separately. As a result of pooling, we identified three SNPs (i.e., CCL5 rs3817655, CCL5 rs2107538, CCR5 rs1799988) that were significantly associated with prostate cancer in the total population even after adjusting for age and multiple hypothesis testing. Upon stratification by study center, we cannot rule out the possibility that race/ethnic specific sequence variants may track with disease progression or prognosis. Consequently, future targeted sequencing will allow us to identify, evaluate and validate novel and commonly reported chemokine-associated SNPs as tumor classification and prognostication tools among African-American, Caribbean, African and European men. Such efforts will require pooled data from multicenter studies that seek to identify the genetic signatures related to prostate cancer health disparities domestically and globally. In addition, molecular biological studies are needed to understand the functional consequence of the CCL5 and CCR5 sequence variants on: mRNA expression/stability, or protein expression, structure and/or function. This will require well annotated observational studies that allow us to consider lifestyle, geographical, environmental, or cultural differences that may interact with genetic susceptibilities and subsequently modify PCA outcomes. In future studies, we will determine the impact of coding and noncoding SNPs on mRNA and protein levels in tissue collected from men of African and European descent using ex vivo, in vitro, and tissue-based assays. Lastly, study participants in the current study self-identified themselves as African-American, Caribbean, African, or Jamaican. Population admixture, which commonly occurs among men of African descent, may bias risk estimates. After adjusting risk estimates for West African Ancestry, risk estimates did not significantly vary when compared to unadjusted risk models (data not shown). In the current study, we had greater than $80 \%$ statistical power to observe effect sizes of $\geq 1.5$ (or $\leq 0.67$ ) and 1.55 (or $\leq 0.64$ ) for the total and U.S. populations, respectively. All p-values in the current study were adjusted for multiple hypotheses testing to minimize false positive results using the false discovery rate. Despite these efforts, we cannot rule out the possibility that the significant relationships observed for the U.S. (CCL5 rs3817655) and total populations (CCL5 rs2107538, CCL5 rs3817655, CCR5 rs1799988) are suggestive and warrant further validation in larger studies. Future studies in our laboratory will focus on high-throughput targeted sequencing to evaluate the impact of novel and commonly reported CCL5 (rs2107538, CCL5 rs3817655) and CCR5 (rs1799988) sequence variants on PCA susceptibility and disease prognosis among men of African descent. Even modest variations in genotype allele frequencies among men of African descent can reduce the chances of replicating single SNP effects within and between independent and validation study sets. To ensure reproducibility within future and ongoing studies, extreme care is needed to select study sets with comparable ancestry and genetic backgrounds.

\section{Conclusions}

Our preliminary findings suggest that CCL5 (rs2107538, rs3817655) and CCR5 (rs1799988) SNPs may modify PCA susceptibility among men of African descent in the current study. In larger ongoing studies, we will evaluate the impact of individual or combination of chemokineassociated SNPs in relation to prostate cancer risk, tumor grade, biochemical or disease recurrence, and mortality. Such efforts will help to identify genetic 
markers capable of explaining disproportionately high prostate cancer incidence, mortality, and morbidity rates among men of African descent.

\section{Additional files}

\section{Additional file 1: Baseline Characteristics among men of African Descent from the US. \\ Additional file 2: Baseline Characteristics among men from Jamaica.}

Additional file 3: Functional Consequence of Chemokine-Associated Sequence Variants ${ }^{a}$.

\section{Abbreviations}

PCA: Prostate cancer; SNP: Single nucleotide polymorphism; CCL: Chemokine ligand; CCR: Chemokine receptor.

\section{Competing interests}

LRK and KSK submitted a patent application for the main study findings mentioned in the manuscript. The authors declare that they have no competing interests.

\section{Authors' contributions}

LRK and KSK: conceptualized the project. LRK, KSK, CR, MJ, NM, MT, SM: participated in the study design. LRK: composed the manuscript. DJ, ER: revised subsequent manuscript drafts. DJ, NCK, SB, JER: conducted quality control and statistical analysis. LRK and GNB: co-supervised quality control analysis, data-management and statistical analysis. LRK, DJ, ER, KSK, SSB: interpreted the data, gave important intellectual input toward the introduction, results and/or discussion. All co-authors: read and edited the manuscript drafts as well as gave final approval of the final manuscript draft.

\section{Acknowledgements}

The authors thank Dr. Rick Kittles for the contribution of DNA samples for this study as well as Tiva T. VanCleave and Nicole A. Lavender for DNA sample preparation. We appreciate the contract services of Expression Analysis, Inc. (http://expressionanalysis.com/) for the generation of genotype data. Grant/Research support: Clinical Translational Science Pilot Grant to LRK the JGBCC Bucks for Brains "Our Highest Potential" in Cancer Research Endowment to LRK; P20-MD000175 NIH NCMHD to KSK.P20-M.

\section{Author details}

'Department of Pharmacology \& Toxicology, University of Louisville Clinical \& Translational Research Building, 505 South Hancock Street Room 306, Louisville, KY 40202, USA. ²Biomedical/Biotechnology Research Institute (BBRI), North Carolina Central University, 531 Nelson Street, Durham, NC 27707, USA. ${ }^{3}$ Cancer Prevention and Control Program, Fox Chase Cance Center, 333 Cottman Avenue, Philadelphia, PA 19111, USA. ${ }^{4}$ Department of Community Health and Psychiatry, University of West Indies, Ring Road, Mona, Jamaica. ${ }^{5}$ Department of Bioinformatics \& Biostatistics, University of Louisville, School of Public Health \& Information Sciences, 485 East Gray Street, Louisville, KY 40202, USA. ${ }^{6}$ School of Medicine, University of Louisville, 323 East Chestnut Street, Louisville, KY 40292, USA.

Received: 9 October 2012 Accepted: 29 October 2012

Published: 20 November 2012

\section{References}

1. American Cancer S: Cancer Facts and Figures 2012. Atlanta, Georgia: American Cancer Society; 2012

2. American Cancer S: Cancer Facts \& Figures for African Americans 2011-2012. Atlanta: American Cancer Society; 2011.

3. Ferlay J, Shin HR, Bray F, Forman D, Mathers C, Parkin DM: Estimates of worldwide burden of cancer in 2008: GLOBOCAN 2008. Int J Canc 2010, 127(12):2893-2917

4. Luster AD: Chemokines-chemotactic cytokines that mediate inflammation. N Eng J Med 1998, 338(7):436-445.

5. Rollins BJ: Chemokines. Blood 1997, 90(3):909-928.
6. Strieter RM, Burdick MD, Gomperts BN, Belperio JA, Keane MP: CXC chemokines in angiogenesis. Cytokine Growth Factor Rev 2005, 16(6):593-609.

7. Strieter RM, Polverini PJ, Kunkel SL, Arenberg DA, Burdick MD, Kasper J, Dzuiba J, Van Damme J, Walz A, Marriott D, et al: The functional role of the ELR motif in CXC chemokine-mediated angiogenesis. J Biol Chem 1995, 270(45):27348-27357

8. Chang CC, Chen SC, Hsieh YH, Chen YC, Chen TY, Chu YH, Ma HJ, Chou MC, Tsai HT, Yang SF: Stromal cell-derived factor-1 but not its receptor, CXCR4, gene variants increase susceptibility and pathological development of hepatocellular carcinoma. Clin Chem Lab Med 2009, 47(4):412-418.

9. Hirata H, Hinoda $Y$, Kikuno N, Kawamoto K, Dahiya AV, Suehiro $Y$, Tanaka Y, Dahiya R: CXCL12 G801A polymorphism is a risk factor for sporadic prostate cancer susceptibility. Clin Canc Res 2007, 13(17):5056-5062.

10. Liou JM, Lin JT, Huang SP, Wu CY, Wang HP, Lee YC, Chiu HM, Shun $C T$, Lin MT, Wu MS: RANTES-403 polymorphism is associated with reduced risk of gastric cancer in women. J Gastroenterol 2008, 43(2):115-123

11. Vindrieux D, Escobar $P$, Lazennec G: Emerging roles of chemokines in prostate cancer. Endocr Relat Cancer 2009, 16(3):663-673.

12. Soria G, Ben-Baruch A: The CCL5/CCR5 axis in cancer. New York: Human Press; 2009.

13. Vaday GG, Peehl DM, Kadam PA, Lawrence DM: Expression of CCL5 (RANTES) and CCR5 in prostate cancer. Prostate 2006, 66(2):124-134.

14. Coussens LM, Werb Z: Inflammation and cancer. Nature 2002 , 420(6917):860-867.

15. Manes S, Mira E, Colomer R, Montero S, Real LM, Gomez-Mouton C, Jimenez-Baranda S, Garzon A, Lacalle RA, Harshman K, et al: CCR5 expression influences the progression of human breast cancer in a p53-dependent manner. J Exp Med 2003, 198(9):1381-1389.

16. Zhernakova A, Alizadeh BZ, Eerligh P, Hanifi-Moghaddam P, Schloot NC, Diosdado B, Wijmenga C, Roep BO, Koeleman BP: Genetic variants of RANTES are associated with serum RANTES level and protection for type 1 diabetes. Genes Immun 2006, 7(7):544-549.

17. An P, Nelson GW, Wang L, Donfield S, Goedert JJ, Phair J, Vlahov D, Buchbinder S, Farrar WL, Modi W, et al: Modulating influence on HIV/AIDS by interacting RANTES gene variants. Proc Natl Acad Sci U S A 2002, 99(15):10002-10007.

18. Saenz-Lopez P, Carretero R, Cozar JM, Romero JM, Canton J, Vilchez JR, Tallada M, Garrido F, Ruiz-Cabello F: Genetic polymorphisms of RANTES IL1-A, MCP-1 and TNF-A genes in patients with prostate cancer. BMC Cancer 2008, 8:382.

19. Purdue MP, Lan Q, Menashe I, Zheng T, Zhang Y, Yeager M, Hosgood HD 3rd, Zahm SH, Chanock SJ, Rothman N, et al: Variation in innate immunity genes and risk of multiple myeloma. Hematol Oncol 2011, 29(1):42-46

20. Bracci PM, Skibola CF, Conde L, Halperin E, Lightfoot T, Smith A, Paynter RA, Skibola DR, Agana L, Roman E, et al: Chemokine polymorphisms and lymphoma: a pooled analysis. Leuk Lymphoma 2010, 51(3):497-506.

21. Enjuanes A, Benavente $Y$, Bosch F, Martin-Guerrero I, Colomer D, PerezAlvarez S, Reina O, Ardanaz MT, Jares P, Garcia-Orad A, et al: Genetic variants in apoptosis and immunoregulation-related genes are associated with risk of chronic lymphocytic leukemia. Cancer Res 2008 68(24):10178-10186.

22. Weng CJ, Chien MH, Lin CW, Chung TT, Zavras Al, Tsai CM, Chen MK, Yang SF: Effect of CC chemokine ligand 5 and CC chemokine receptor 5 genes polymorphisms on the risk and clinicopathological development of oral cancer. Oral Oncol 2010, 46(10):767-772.

23. Duell EJ, Casella DP, Burk RD, Kelsey KT, Holly EA: Inflammation, genetic polymorphisms in proinflammatory genes TNF-A, RANTES, and CCR5, and risk of pancreatic adenocarcinoma. Canc Epidemiol Biomarkers Prev 2006, 15(4):726-731.

24. Kidd LC, Vancleave $\Pi$, Doll MA, Srivastava DS, Thacker B, Komolafe O, Pihur V, Brock GN, Hein DW: No association between variant Nacetyltransferase genes, cigarette smoking and Prostate Cancer susceptibility among men of African descent. Biomark Cancer 2011, 2011(3):1-13.

25. Jackson MD, Walker SP, Simpson-Smith CM, Lindsay CM, Smith G, McFarlane-Anderson N, Bennett FI, Coard KC, Aiken WD, Tulloch T, et al: 
Associations of whole-blood fatty acids and dietary intakes with prostate cancer in Jamaica. Canc Causes Contr 2012, 23(1):23-33.

26. Manolio TA, Collins FS, Cox NJ, Goldstein DB, Hindorff LA, Hunter DJ, McCarthy MI, Ramos EM, Cardon LR, Chakravarti A, et al: Finding the missing heritability of complex diseases. Nature 2009, 461(7265):747-753.

27. McCarthy Ml, Abecasis GR, Cardon LR, Goldstein DB, Little J, loannidis JP, Hirschhorn JN: Genome-wide association studies for complex traits: consensus, uncertainty and challenges. Nat Rev Genet 2008, 9(5):356-369.

28. Steemers FJ, Gunderson KL: lllumina, Inc. Pharmacogenomics 2005, 6(7):777-782.

29. Giri VN, Egleston B, Ruth K, Uzzo RG, Chen DY, Buyyounouski M, Raysor S, Hooker S, Torres JB, Ramike T, et al: Race, genetic West African ancestry, and prostate cancer prediction by prostate-specific antigen in prospectively screened high-risk men. Cancer PrevRes(Phila Pa) 2009, 2(3):244-250.

30. Tian C, Hinds DA, Shigeta R, Kittles R, Ballinger DG, Seldin MF: A genomewide single-nucleotide-polymorphism panel with high ancestry information for African American admixture mapping. AmJHumGenet 2006, 79(4):640-649.

31. Menashe I, Rosenberg PS, Chen BE: PGA: power calculator for case-control genetic association analyses. BMC Genet 2008, 9:36.

32. Konig JE, Senge T, Allhoff EP, Konig W: Analysis of the inflammatory network in benign prostate hyperplasia and prostate cancer. Prostate 2004, 58(2):121-129.

33. Petersen DC, Severi G, Hoang HN, Padilla EJ, Southey MC, English DR, Hopper JL, Giles GG, Hayes VM: No association between common chemokine and chemokine receptor gene variants and prostate cancer risk. Canc Epidemiol Biomarkers Prev 2008, 17(12):3615-3617.

34. Blanpain C, Buser R, Power CA, Edgerton M, Buchanan C, Mack M, Simmons G, Clapham PR, Parmentier M, Proudfoot AE: A chimeric MIP-1alpha/ RANTES protein demonstrates the use of different regions of the RANTES protein to bind and activate its receptors. J Leukoc Biol 2001, 69(6):977-985.

35. Fellay J, Ge D, Shianna KV, Colombo S, Ledergerber B, Cirulli ET, Urban TJ, Zhang K, Gumbs CE, Smith JP, et al: Common genetic variation and the control of HIV-1 in humans. PLoS Genet 2009, 5(12):e1000791.

doi:10.1186/1897-4287-10-16

Cite this article as: Kidd et al:: Chemokine Ligand 5 (CCL5) and

chemokine receptor (CCR5) genetic variants and prostate cancer risk among men of African Descent: a case-control study. Hereditary Cancer in Clinical Practice 2012 10:16.

\section{Submit your next manuscript to BioMed Central and take full advantage of:}

- Convenient online submission

- Thorough peer review

- No space constraints or color figure charges

- Immediate publication on acceptance

- Inclusion in PubMed, CAS, Scopus and Google Scholar

- Research which is freely available for redistribution 